\title{
A sensorimotor learning framework for object categorization
}

\author{
Virgile Högman, Graduate Student Member, IEEE, Mårten Björkman, Atsuto Maki, Member, IEEE, \\ and Danica Kragic, Member, IEEE
}

\begin{abstract}
This paper presents a framework that enables a robot to discover various object categories through interaction. The categories are described using action-effect relations, i.e. sensorimotor contingencies rather than more static shape or appearance representation. The framework provides a functionality to classify objects and the resulting categories, associating a class with a specific module. We demonstrate the performance of the framework by studying a pushing behavior in robots, encoding the sensorimotor contingencies and their predictability with Gaussian Processes. We show how entropy-based action selection can improve object classification and how functional categories emerge from the similarities of effects observed among the objects. We also show how a multidimensional action space can be realized by parameterizing pushing using both position and velocity.
\end{abstract}

Index Terms-sensorimotor learning, object classification, categorization, cognitive robotics, active perception, learning and adaptive system, embodiment, developmental robotics.

\section{INTRODUCTION}

Psychological studies show that categorization of objects in humans derives from how we act on them [1]. For a robot, such a capability may enable adaptive behavior, resulting in faster learning of novel objects. Traditionally in robotics, perception and action have been treated separately, commonly designed as a causal system where the relations between perception and action are represented as predefined sets of rules. Object and action categorization have been studied extensively in robotics, computer vision and machine learning communities [2]-[7], but mostly considered as a passive observation problem using prerecorded databases representative for the problem. In robotics, we experience a growing interest in regarding categorization as the result of an active interaction between a robot and the environment [8]-[13]. A robot should acquire categories from its own sensorimotor experiences in a bottom-up fashion.

The studies of O'Regan and Noë [14] put forward a theory that visual consciousness is the result of an action-based exploration of the world, claiming that seeing is a way of acting. This theory further suggests that the outer world can be probed as an external memory by so-called sensorimotor contingencies (SMCs), instead of storing a structured representation. Various levels of knowledge are constituted by associating sensory outcomes with actions and their predictability is learned from experience. In particular, SMCs can be defined

Virgile Högman, Mårten Björkman, Atsuto Maki, Danica Kragic are with the Centre for Autonomous Systems, Computer Vision and Active Perception Lab, CSC, KTH Royal Institute of Technology, Stockholm, Sweden e-mail: ( $\{$ virgile|celle|atsuto|dani\}akth.se.

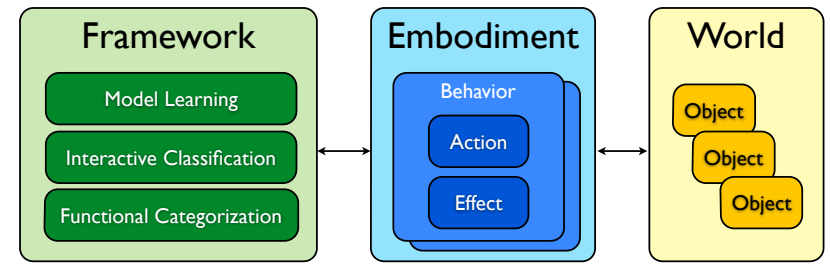

Fig. 1. Our framework for sensorimotor learning aims to classify and categorize objects solely from action. It provides a template for any behavior involving an interaction with objects (e.g. pushing). How the action is performed and its resulting effects are modelled by sensorimotor contingencies which are intrinsic to the robot's embodiment.

for objects, by observing effects that result from interaction through exploratory behaviors [12]-[17]. In this regard they are tightly connected to the notion of affordance [10], [18][23] enabling also the development of tool use [24]-[26].

In this article our interest is to understand how perceptual categories can emerge from SMCs for a given embodiment. We study how a robot can classify objects through interaction and how to ground the categories in its own experience with account for uncertainty and generalization. For this we propose a framework whose outline is illustrated in Fig. 1. We adopt a modular approach based on the MOSAIC model [27], [28]. This model provides a relevant background for sensorimotor learning with training from self-experience and enables prediction. We use a similar representation by associating a module with a class which is an object instance or a category.

Our framework is designed to be generic for any behavior as long as a suitable representation of an action and its effects can be defined. The conditions under which this holds are related to the frame-of-reference problem [29] and are specified later on. To illustrate our concept we study a pushing behavior where translational and rotational features are learned. Independently of the behavior, the contingencies are modelled as object-specific functions:

$$
f_{\Delta}: a \rightarrow \Delta\left(s, s^{\prime}\right),
$$

where $\Delta\left(s, s^{\prime}\right)$ is the sensory change between a set of attributes $s$ prior to action $a$ and the set $s^{\prime}$ afterwards, encoding an effect explicitly. Based on our preliminary work [30] this representation is a variant of a forward model [31] that we learn as distributions using Gaussian Processes (GPs) [32]. What we designate as functional categories are then extracted by measuring the similarities of the observed effects among the different objects.

Moreover we aim to emphasize the constitutive role of 
action and derive an efficient policy for the task of classification. Many studies on action-grounded learning rely on a specific controller and describe actions as high-level behaviors without specifying their parameters [10], [12], [19]. In [30] we defined a pushing action by the contact position on the one-dimensional horizontal axis. In this study we consider a multi-dimensional action space incorporating the velocity of the push and deal with a larger set of objects.

The contributions of the work presented here are:

- A generic framework for sensorimotor learning from interaction with objects, based on the SMCs theory and following a modular approach as in the MOSAIC model.

- Modelling the effects in an object-centered representation and illustrating with a pushing behavior how these are learned with GPs.

- Showing how object classification can be performed from action exclusively and how to exploit confidence in predictions with entropy-based action selection.

- Grounding the categories in the observed effects with account for uncertainty, from the similarities between different GPs.

This paper is organized as follows. Section II presents the theoretical background supporting our approach. Section III reports the related works. Section IV describes our framework and Section $\mathrm{V}$ shows how it can be instantiated by modelling a pushing behavior. Section VI reviews experimental results, followed by conclusions and directions of future works.

\section{BACKGROUND}

In the previous section, we described the concept of sensorimotor contingencies (SMCs) defined as law-like relations between actions and their effects. The SMCs theory [14] suggests that these can be captured at different levels of complexity:

- Modality-related SMCs capture the specific changes of the sensory signal in a single modality depending on the agent's action.

- Object-related SMCs concern the effects on the sensory system that are specific to the objects being acted upon.

- Intention-related SMCs consider the long-term correlation structure between complex actions/action sequences and the resulting outcomes or rewards which the agent learns to predict.

Here we study the second level that corresponds to objects. The SMCs theory does not provide a model to represent the contingencies, but we adopt its principles based on the predictability of actions.

With regard to developmental robotics, Stoytchev [33] formulates five principles to be considered in a learning system. Two of these, verification and grounding, are particularly relevant to our work and support the SMCs theory. The verification principle states that an agent can create and maintain knowledge only to the extent that it can verify by itself. Thus, we avoid any representations other than those derived from observations of action-effect pairs, as illustrated by the example used in this paper in Fig. 2. The principle of grounding sets the boundaries to the concept of verification,

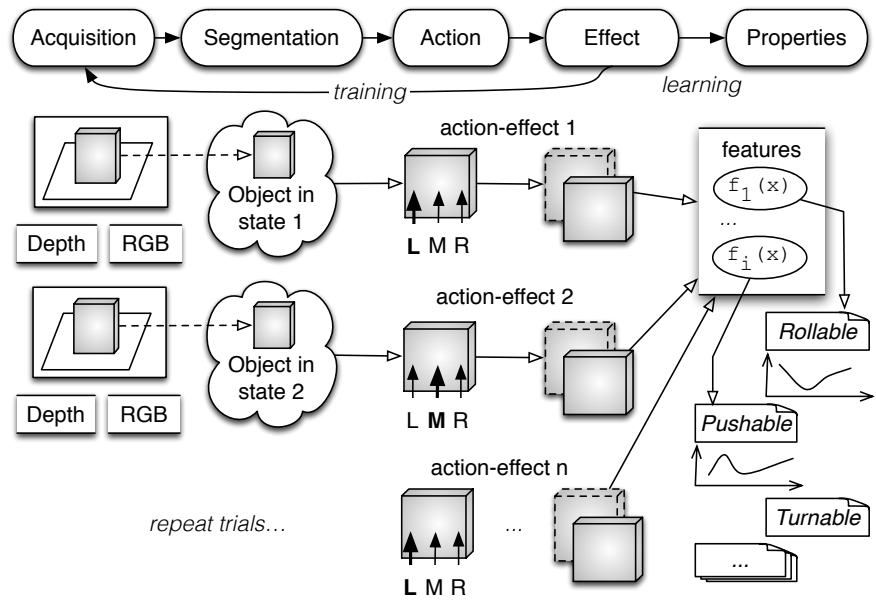

Fig. 2. Learning process: every action applied on the object leads to an effect, illustrated here for a pushing behavior. By repeating actions several times, the agent gains experience that is used for learning of different object properties.

which can be achieved by repeating actions in the same context to gain confidence about the outcomes. Our learning process follows this concept as the employed GPs are trained on sets of observations with the variance measuring the associated uncertainty. This allows us to deal with noise in sensory data or due to the nature of the objects.

Our framework also refers to the MOSAIC model which allows sensorimotor learning and stands for Modular Selection And Identification for Control. Initially proposed as pairs of forward and inverse models [27], it was extended by Haruno et al. [28] with improvements in the learning process. This model is designed from a control point of view and does not concern classification or categorization which is considered in our work. As stated by the authors, it is based on several modules that contain pairs of forward (predictor) and inverse (controller) models [31]. A module represents a particular context that captures the properties of an object in a specific environment. Sensory information about this context is given by a contextual signal, encoding for instance the perceived shape of object. Each module is defined by three components:

- a predictor model evaluates the prior information from the contextual signal, before performing the action,

- a forward model, combined with a likelihood model of the prediction error (difference between the actual and predicted states), provides a feedback after performing the action,

- an inverse model gives the action control necessary to achieve a specific goal defined by a target state.

From the predictor and the forward models, the responsibility of each module is evaluated. It represents how well a module corresponds to both prediction and observation of the actual object under consideration, formulated as follows:

$$
\lambda_{t}^{i}=\frac{\pi_{t}^{i} l_{t}^{i}}{\sum \pi_{t}^{j} l_{t}^{j}}
$$

where $\pi_{t}^{i}$ and $l_{t}^{i}$ are respectively the priors and likelihood of the $i^{t h}$ module at time $t$. After a phase of adaptation, each responsibility reflects a specific context. To reach a desired 
state, the responses of each module are combined with their respective responsibility so that the final output is a weighted sum of the individual inverse models.

Within our framework we adopt a similar modular and probabilistic approach, where each module represents a class (object or category) that contains the forward model together with the likelihood model. Though the prior is defined differently and the modules are trained separately, the resulting responsibility is computed in a similar manner. The main difference between the two frameworks comes from the task to achieve. Unlike MOSAIC, our task of classification does not involve a target state. The responsibility is not used for the control of the action but it corresponds to the posterior used for classification.

Following the SMCs theory we study how classification can be performed if exclusively driven by action. For this reason we do not consider the contextual signal to evaluate a prediction before an action, though it could be added as a prior. Instead, an action selection component provides the next action to take in sequence. The context associated with each SMC corresponds to the instance of the object. The learning is supervised, meaning the instance is known during the learning stage, and our experiments are performed in a stable environment which is not modelled explicitly. For example we do not take into account the surface of support, obstacles, or deformable objects.

Finally, categorization is defined as a global component over the modules representing the classes. We consider it as a problem of unsupervised learning given the effects or in other words a problem of clustering provided the likelihood models.

\section{RELATED WORK}

In terms of related work, a parallel can be drawn to ObjectAction Complexes (OACs) [34]. OACs have been proposed as building blocks in a cognitive architecture and are formalized to include an object and action specific transition function:

$$
T: s \rightarrow s^{\prime} .
$$

OACs are organized in different levels of abstraction, where low-level OACs can be loosely mapped to modality-related SMCs and high-level OACs to intention-related ones. Close to this concept, object-action relations are represented by scene graphs in [35] after observing sequences of actions described at high level. Objects are categorized by measuring similarities in the spatiotemporal domain among semantic event chains. Our framework instead shows how to drive the sequence of actions to recognize objects or categories from interaction.

Also related to our work, the Distributed Adaptive Control (DAC) [36] presents a biologically inspired model, with a three-layered architecture. A reactive layer provides a prewired set of reflexive behaviors; an adaptive layer allows adaptive classification of sensory events; and a contextual layer uses long-term and short-term memory to support action sequences. It can be formalized as:

$$
f:\{a, s\} \rightarrow a^{\prime}
$$

where $\{a, s\}$ is a sequence of action-outcome pairs and $a^{\prime}$ is a new action for the robot to pursue. Thus, unlike the forward model in OACs where the function gives predicted sensory data after an action, DAC uses an inverse model [31].

Several publications refer to sensorimotor learning from interaction with objects. The work reported in [19], [37] use pushing to learn object affordance of "rollability". The learned effect is defined as the probability of success to roll or not. We do not assume the agent knows how to evaluate the resulting objects properties and preserve the effect as sensory information with translation and rotation features.

Affordance gradients are introduced in [20] to model how object's rotation and translation change as it is pushed. The shape is learned from IR readings of a small E-puck robot that is circulating around the object. A similar setup is used in [38] but lets sensory and action spaces be discretized with earlier observations recorded as SMCs stored in histograms. Our model instead allows continuous sensory and action spaces; and, to learn object-related SMCs from a more limited set of pushes using a real robotic arm, we represent these as Gaussian Processes (GPs) from which predictions can be made already after a few exploratory trials.

Similar to our work, categories are discovered through unsupervised clustering in the effect space [8]-[11], [13], [39] though only used for predictions in [10]. Different modalities are covered such as vision, audio, proprioception, vibrotactile, alone or combined with different behaviors, applied to a large range of objects [12] though the categories were known in this case. However these works show a limited account for uncertainty and generalization, depending tightly on the quantity and quality of training data with rich features. SMCs are mostly represented by sets of tuples from training observations, to learn for instance empirical distributions or discriminative models. Our model gives the robot the ability to make predictions and evaluate the confidence of action outcomes, which is exploited in classification similar to [12], [16]. In contrast, our action selection is driven by a measure of entropy and the categories are discovered from the similarities of the learned GPs. Though we demonstrate this concept for only vision and one behavior, the components of the framework are generic and fully grounded by experience. In [15] entropy is used to find a policy for action selection through reinforcement learning. Instead we exploit it directly from the actual estimates before taking each action.

Also performing a pushing behavior, the common approach to the works reported in [9], [10], [12], [39] is to define a high dimensional feature space from the perceptual data, including a representation of the shape. In contrast, our work focuses on the action-effect relation with an explicit representation of the effect. Shape is represented implicitly by the effects the shape gives rise to. Our feature space with pushing is limited to translation and rotation but it is restricted intentionally to study generalization from low-level effects. Classification is done not from predictions using the sensory data alone, but as a result from multiple observations of effects from selected actions applied to objects. Though passive observations are ignored, experiments show that categories of various shapes can still be recognized. 


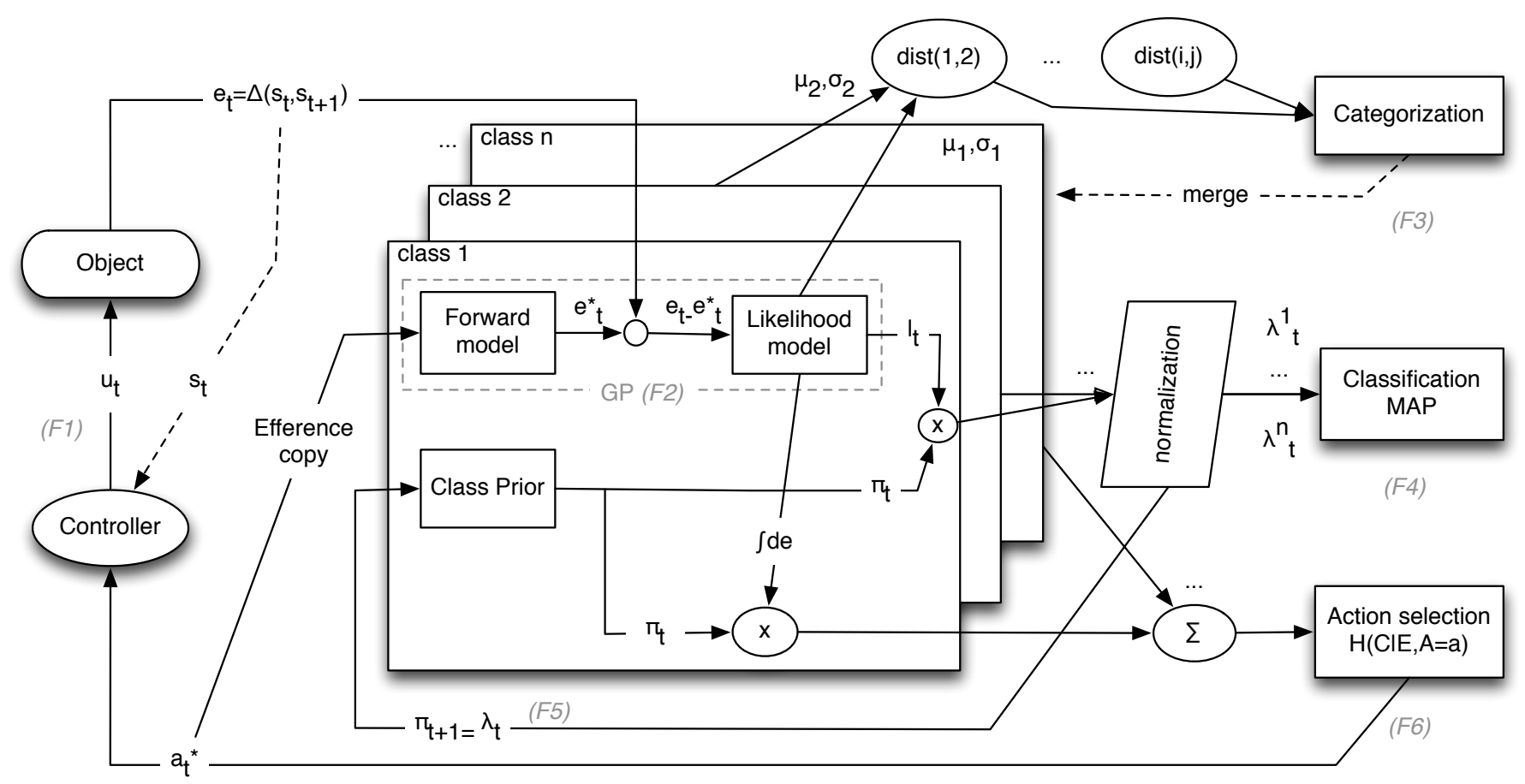

Fig. 3. Our framework for sensorimotor learning, adopting a modular approach for interactive classification and functional categorization of objects.

\section{OUR FRAMEWORK}

In this section we propose a new framework based on object-related SMCs illustrated in Fig. 3. We start by defining the terms used in the following parts of the paper:

- Behavior: type of performance e.g. push, grasp, drop, squeeze.

- Action: specific behavior defined by a parametrization indicating how the performance is done e.g. push left with a given angle and speed.

- Effects, outcome: result of an individual action taken on an object, measured with perceptual features.

- Sensorimotor contingency (SMC): relation between an action and its effects, for a given class (object instance or category).

\section{A. Action-effect as forward model}

Through the observation of object-related SMCs, we study the effects of different actions on an object and their predictability. From a control perspective, an action applied to an object leads to a change of its state. This can be represented by a forward model [31], providing the prediction of the state $s^{\prime}$ after applying an action $a$ on the object in state $s$ :

$$
f: s, a \rightarrow s^{\prime} .
$$

By observing the change of states from $s$ to $s^{\prime}$, respectively before and after action, we define an effect as $\Delta\left(s, s^{\prime}\right)$ where the function $\Delta$ is an effect feature. Assuming that sensory information is always measured with respect to an object reference, as done in [30], we reformulate the function (2) as follows:

$$
f_{\Delta}: a \rightarrow \Delta\left(s, s^{\prime}\right)
$$

where $a$ defines the action and $\Delta\left(s, s^{\prime}\right)$ represents an explicit effect, depending on the chosen features. For example, with the pushing behavior presented in the next section, a translation effect is defined as a function of the position of the object. It is computed as the Euclidean distance between the object positions measured before and after push. For clarity, the effect is denoted by $e=\Delta\left(s, s^{\prime}\right)$ in the following parts.

In contrast to the general form of the forward model (2) the action $a$ taken in (3) is independent of the state $s$. This is allowed by the object-centered representation that we describe further for the pushing behavior. This also means the action space $a$ is a symbolic representation in the object frame. To apply the action $a$ to the object in the physical world, a controller translates $a$ into motor commands $u$, given the state of the object $s$ at time $t$ as shown in Fig. 3 (F1).

\section{B. Learning with Gaussian Process}

Following the principle of grounding [33], an agent acquires knowledge with a certain degree of confidence from repeated observations of action-effect pairs. This can be achieved with a probabilistic representation through statistical learning, where the distribution of the effect $e$ given action $a$ is learned as a probability $p(e \mid a)$. This distribution englobes both the forward and likelihood models as illustrated in $(F 2)$.

We base our model on Gaussian Process regression (GP) [32] where the variance takes both the current lack of observations and the noise in outcomes into account. In other terms the variance represents the confidence the robot has over its SMC. This approach also allows us to infer on a continuous action space though the experiments are limited to a discrete case. We decompose $f_{\Delta}$ such that each feature is represented by a GP, defined by a mean and a covariance 
function, with additive white noise. We use a zero-mean Gaussian, with covariance defined by the squared exponential with Automatic Relevance Determination (ARD) distance measure to cope with different dimensions in the action space. The optimal hyperparameters are found by optimizing the marginal likelihood of the training data as described in [32].

Another motivation for choosing the GP is that a similarity measure used for categorization $(F 3)$ can be derived naturally from the means $\mu$ and variances $\sigma^{2}$ related to each class. In the experiments we propose a symmetric distance measure based on the Kullback-Leibler divergence between two classes.

\section{Interactive classification}

Once object-related SMCs have been learned for a given number of classes (object instances or categories), through the training of the GP, the agent can perform functional classification on new objects. By taking an action $a$ and observing its effect $e$, it is possible to determine which class the object is most likely to belong to. Assuming the classes of the training samples are known, a Bayes classifier (F4) is defined as follows:

$$
p(c \mid e, a)=\frac{p(e \mid c, a) p(c)}{p(e \mid a)}=\frac{p(e \mid c, a) p(c)}{\sum_{c^{\prime}} p\left(e \mid c^{\prime}, a\right) p\left(c^{\prime}\right)},
$$

where $c$ is the object class, $e$ is the observed effect after executing the action $a$, and $p(c)$ the class prior that is independent on the action (uniform distribution in the general case). The likelihood $p(e \mid c, a)$ is given by the GP previously learned for each class. For clarity, (4) is equivalent to (1) after specifying the terms as: $p(c)=\pi ; p(e \mid c, a)=l ; p(c \mid e, a)=\lambda$. The estimated object class is then determined by the maximum a posteriori probability (MAP):

$$
c^{*}=\arg \max _{c} p(c \mid e, a) .
$$

Effects may be composed of $F$ different features $e_{1} \ldots e_{F}$. In our experiments, we have two; $e_{1}$ is the translation and $e_{2}$ is the rotation. If we assume these random variables to be independent, as done in the experiments, these can be combined by multiplication (naive Bayes classifier):

$$
p\left(c \mid e_{1}, e_{2}, \ldots e_{F}, a\right)=\frac{\prod_{i=1}^{F} p\left(e_{i} \mid c, a\right) p(c)}{\sum_{c^{\prime}} \prod_{i=1}^{F} p\left(e_{i} \mid c^{\prime}, a\right) p\left(c^{\prime}\right)} .
$$

Observations from several actions can also be combined by multiplying all the probabilities of the sequence. We expect that the more actions we take, the higher the confidence we get by making more observations. To proceed with a sequence of actions, the resulting classification after the execution of an action at time step $t$ can be used as the new prior of each class (F5) in the next step $t+1$. Equation (4) can then be rewritten as:

$$
p_{t}(c \mid e, a)=\frac{p_{t}(e \mid c, a) p_{t}(c)}{p_{t}(e, a)}
$$

where $p_{t}(c)=p_{t-1}(c \mid e, a)$ and the initial prior $p_{0}(c)$ is a uniform distribution. At every time step, the features are combined as described in (6).

\section{Action selection}

A new object is classified by observing the effects of an action or multiple actions in sequence. To determine which action should be taken, one can look at the outcome distributions of the known classes. As the outcome is not known in advance for a new object, it is preferable to choose the action that will discriminate the classes with the highest confidence, or in other terms, the action that provides the highest expected information gain. This can be seen as if the agent knew the most appropriate action for this purpose, given its experience represented by the SMCs. Based on information theory, we use the entropy of the class distributions as a criterion to measure the uncertainty related to each action. By looking for the action associated with the lowest predicted entropy, we can select the one that is likely to classify the object with the highest confidence.

In our case, we look for the class given by the highest probability of $p(C \mid E, A=a)$ for action $a$, where $C$ is a discrete random variable for the class and $E$ is a random variable corresponding to possible effects. Therefore, we measure the conditional entropy of $C$ given $E$, for the action $a$ :

$$
H(C \mid E, A=a)=-\int \sum_{c} p(c, e \mid a) \log p(c \mid e, a) \mathrm{d} e .
$$

Using Bayes' rule in the joint distribution, and rearranging the terms, we reformulate this equation as follows:

$$
\begin{aligned}
H(C \mid E, A=a) & =-\int \sum_{c} p(e \mid c, a) p(c) \log p(c \mid e, a) \mathrm{d} e \\
& =-\sum_{c} p(c) \int \log p(c \mid e, a) p(e \mid c, a) \mathrm{d} e .
\end{aligned}
$$

As the likelihood $p(e \mid c, a)$ has been learned with the GPs, the integral can be approximated with a Monte Carlo method, drawing a finite set of samples from each class distribution. The result is normalized with the total number of samples $N(N=100$ in the experiments):

$$
\hat{H}(C \mid E, A=a)=-\frac{1}{N} \sum_{c} p(c) \sum_{k=1}^{N} \log p\left(c \mid e_{k}^{c}, a\right),
$$

where $p\left(c \mid e_{k}, a\right)$ can be computed with Bayes' rule as defined in (4), using the likelihood $p\left(e_{k}^{c} \mid c, a\right)$ normalized over all the classes, each $e_{k}^{c}$ being sampled from the distribution for a given class $c$ and action $a$. To combine several features, we use the variant defined in (6). Finally, we can select the best action (F6) as the one resulting in the lowest entropy:

$$
a^{*}=\underset{a}{\arg \min } \hat{H}(C \mid E, A=a) \text {. }
$$

At every time step the best action is evaluated, and the cumulated posterior probability of each class is then updated with (7). Similarly, these posterior probabilities are used as class priors in (9) for the next time step.

\section{E. Summary}

We have presented a framework for sensorimotor learning, functional categorization and interactive classification with 
entropy-based action selection. This template can be instantiated for a behavior, where action-effects are defined in an object-centered representation. What remains to be defined is the action-effect feature space and how to translate it in the physical world.

\section{A MODEL FOR PUSHING}

To illustrate the concepts presented so far we study how objects of different shape behave after a pushing behavior has been applied to them. We model translation and rotation effects in terms of object-related SMCs.

Two low-level processes are assumed to exist: a foveation process that directs the gaze towards an object of interest, keeping it in the center of view, and a perceptual grouping process that segments the object from its surroundings. Thus, a reference frame can be established with the object centroid as its origin. We consider this a low-level function the robot has already learned (modality-related SMCs).
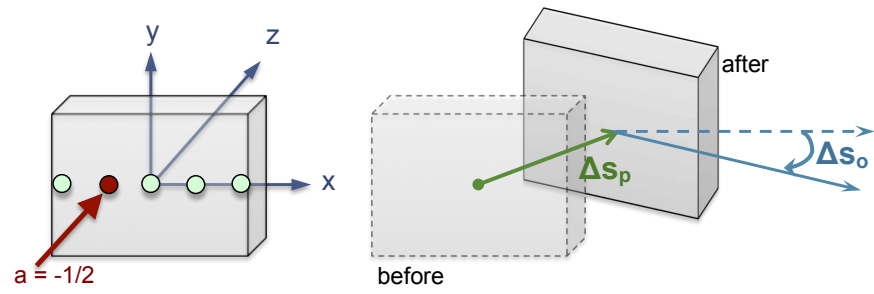

Fig. 4. Effect of a push, described by translation and rotation features, in an object-centered representation. The dots along the $\mathrm{X}$-axis represent the possible actions used in the experiments.

We adopt an object-centered representation where a push is generated with respect to the local object frame. A dominating plane, which defines this frame, is sought by fitting a plane to the 3D point cloud representing the segmented object region, a procedure that is done with RANSAC [40] in the experiments. Note that for many objects, such as balls, the extracted dominating plane will not correspond to a real physical plane on the object itself, but be a virtual plane to which the highest number of 3D points belong. Once the object orientation is determined, a local coordinate system is defined as shown in Fig. 4. We consider objects lying on a horizontal plane such as a table, so the push is always performed along the $Z$-axis. Coordinates are normalized along the $X$ - and $Y$-axes, such that -1 and +1 correspond to the extent of the object on either side.

A pushing action is defined by $a=\left(a_{X}, a_{V}\right)$ where $a_{X}$ is the position on the extracted dominating plane measured along the $X$-axis and $a_{V}$ is the velocity of the push. In the experiments we kept the height fixed to positions along the lower part of the object, such as the objects do not fall, and applied actions in directions parallel to the support where the object is placed. The velocity $a_{V}$ extends the dimensionality of the action space previously defined by $a=\left(a_{X}\right)$ in [30]. Some object properties such as rolling for the balls, are only "triggered" from a given velocity. If the ball is pushed too slowly, it is more likely to slide and be assimilated to the other objects. To compare the sole effect of the velocity in relation to the translation feature, the distance for which the push is applied is kept constant in the experiments. Thus $a_{V}$ describes a symbolic velocity translated in motor commands as $\left(u_{\text {speed }}, u_{\text {duration }}\right)$, in the same way as $a_{X}$ is a relative position in the object-centered representation.

As mentioned in (3), an SMC can be expressed as a function:

$$
f_{\Delta}: a \rightarrow \Delta\left(s, s^{\prime}\right),
$$

where $e=\Delta\left(s, s^{\prime}\right)$ is an effect, depending on the states $s$ and $s^{\prime}$ before and after the pushing action $a$. We define the functions $\left(\Delta_{p}, \Delta_{o}\right)$ as the translation and rotation effects respectively. We measure each state through $s_{p}$ the position of the foveated object and $s_{o}$ the orientation of the dominating plane. From the segmented point cloud, we have a vector $s_{p}=\left(x_{p}, y_{p}, z_{p}\right)^{T}$ for the 3D centroid, and from the plane we have $s_{o}=\left(x_{o}, y_{o}, z_{o}\right)^{T}$ describing the normal vector of this plane. The translation is computed as the Euclidean distance of the change in position. Hence we have $\Delta_{p}\left(s, s^{\prime}\right)=\left\|s_{p}^{\prime}-s_{p}\right\|$ where $s_{p}$ and $s_{p}^{\prime}$ are the positions before and after push. The rotation is the dihedral angle between the two planes, so that $\Delta_{o}\left(s, s^{\prime}\right)=\angle\left(s_{o}, s_{o}^{\prime}\right)$ where $s_{o}$ and $s_{o}^{\prime}$ are the corresponding normal vectors.

\section{EXPERIMENTS}

After presenting the experimental setup, we review results from classification of the object instances, with entropy-based action selection, followed by unsupervised categorization.

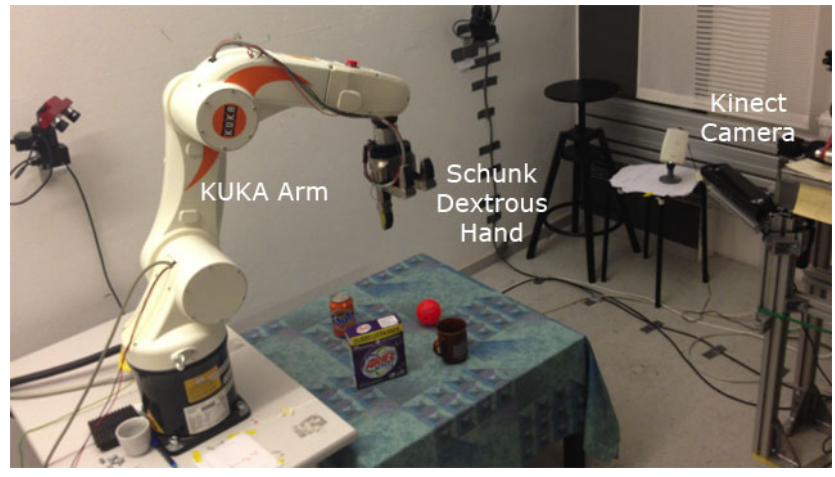

Fig. 5. Setup with Kuka Arm and Schunk Hand. The Kinect camera is fixed and points towards the table where the objects lie.
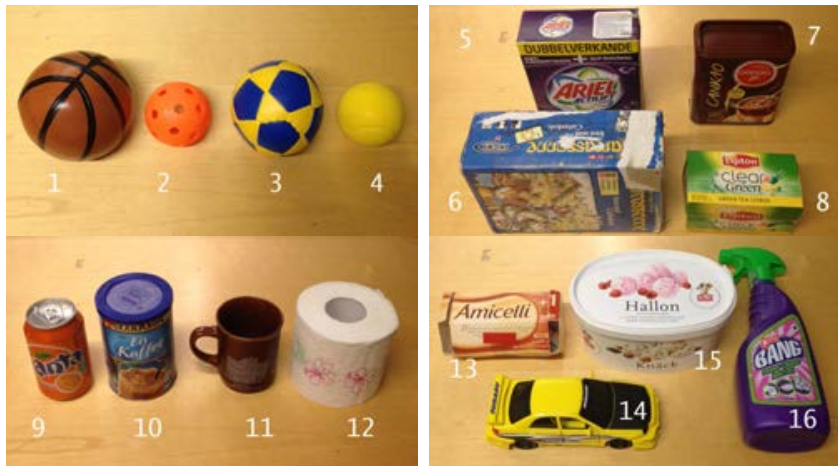

Fig. 6. The 16 objects considered in the experiments, with four instances for each of the four groups: balls, boxes, cylinders and miscellaneous. 


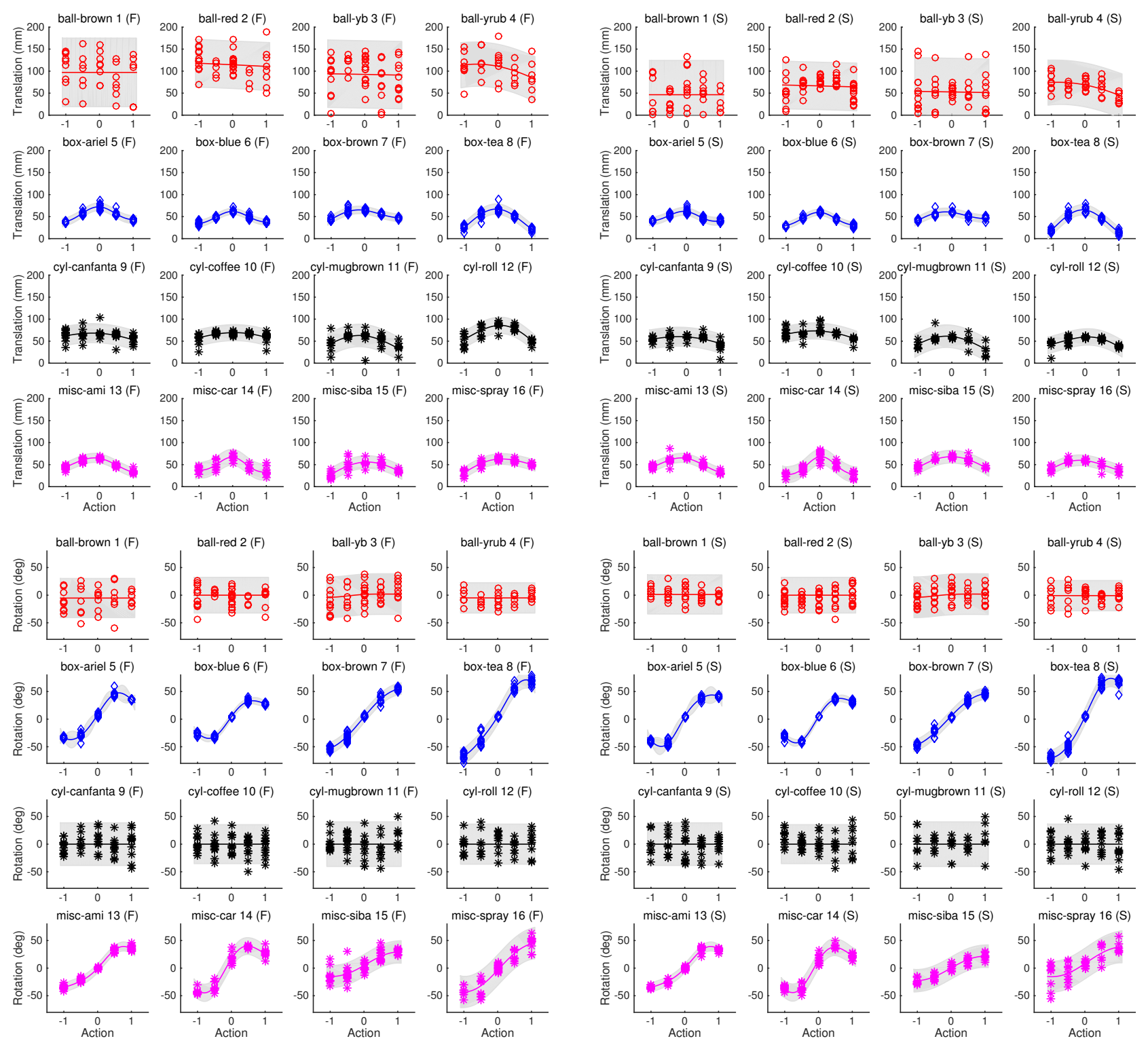

Fig. 7. Learned function of translation (top) and rotation (bottom) for each object, given action as a Fast push (left side) or Slow push (right side). The X-axis represents the push position $a_{X}$. Each row corresponds to a different group: ball, box, cylinder, miscellaneous. The marks correspond to the samples of the dataset. The mean and variance of the GPs are described respectively by the lines and the grey areas with a $2 \sigma$ deviation, representing $95 \%$ of confidence. The main difference noticeable between the fast and slow push comes from a lower mean for the translation of the balls when pushed slowly.

\section{A. Setup}

In the experiments objects of different shapes are placed on a table and pushed one by one with a robotic configuration (Kuka Arm and Schunk Hand) shown in Fig. 5. The effects are observed by a fixed Kinect camera, providing color and depth information. A total of 16 different objects (see Fig. 6) are considered, organized in four groups (balls, boxes, cylinders and miscellaneous) of four objects each. The miscellaneous group contains objects which present different shapes from the three main groups.

The object specific sensory data from which the effect of actions are studied are extracted as follows. First an object is segmented from the image using object segmentation [41]. From color and depth data, a 3D point cloud is built from which the center of mass of the object is estimated. However, only the visible part of the object is taken into account, which means that the center is likely to be shifted.

The learning phase consists of pushing the objects horizontally at different locations and velocities given by $\left(a_{X}, a_{V}\right)$. The model allows a continuous action space, but is discretized here in order to separate the noise level in outcomes from the variability of the modelled functions in the interpretation of the data. The position $a_{X}$ takes values in the discrete set $\{-1,-1 / 2,0,+1 / 2,+1\}$ as shown in Fig. 4. The vertical position is kept constant at $1 / 3$ of the height of the object. The velocity $a_{V}$ is either slow or fast, represented by the set $\{1,2\}$. The combination of $\left(a_{X}, a_{V}\right)$ gives 10 possible actions.

To determine the position in the arm frame from the relative offset $a_{X}$ in the object frame, the width of the object is estimated from a thin slice of the object cloud at $+/-1 \mathrm{~cm}$ around the pushing height. The height of the object is estimated from 
the extreme $Y$-values of the cloud. The orientation of the push is given by the most dominant plane, such that the push is performed along the normal of this plane. If the plane is not found with sufficient confidence, the orientation for the push is instead estimated by Principal Component Analysis (PCA) using the implementation in OpenCV based on the KarhunenLoeve Transform. A thin slice of the object cloud is extracted at $+/-1 \mathrm{~cm}$ around the pushing height. The $1 \mathrm{st}$ eigen vector of the covariance matrix of these points corresponds to the dominant orientation of the object at that height, and the 2 nd eigen vector gives the direction of the push.

In our preliminary experiments [30] the states before and after push were loosely defined. The duration of the push varied with the position of the push and the shape of the object. To observe more regular effects, the push is now decomposed in two steps: an initial approach and the effective push, which starts from the moment the object begins to move. To detect the motion of the object, the segmentation process is combined with the dense optical flow using Farnebäck's algorithm [42] in OpenCV. The state after push is defined about $1 s$ after completing the push and lifting up the arm.

\section{B. Gaussian Process models}

After pushing each object repeatedly, exploring randomly the 5 locations and 2 different velocities in the training stage, a dataset with 1674 samples is compiled for analysis, representing an average of 10 pushes per object and action (848 fast and 813 slow pushes, between 78 and 138 pushes per object). Fig. 7 illustrates the learned functions of translation and rotation for each object, given different actions $a$.

As expected, the balls roll with a higher amplitude than the other objects that slide a shorter distance. The rotation feature is only relevant for the boxes and the miscellaneous objects, leading to a small variance, while its variance is much higher for the other objects. This can be explained by the detection of the plane, which is not suitable for spherical or cylindrical objects. However, this characteristic is captured by the variance, which is exploited subsequently for classification after multiple pushes. The boxes rotate with different amplitudes, depending mostly on the weight of the object. For the observed action effects, the miscellaneous objects look mostly like boxes. To what degree they can be categorized to boxes is studied in further experiments.

\section{Classification with action selection}

By applying a sequence of pushes to an object, the probability it belongs to each class is estimated with the cumulated posteriors (7). To verify if the entropy-based action selection policy (MinEnt) improves classification, we compare this policy where the action is given by (10) to randomly selected actions (Random). Since the dataset is limited in the number of samples, we proceed with 2-fold cross-validation, generating two separate sets of equal size for training and test. Each fold is tested over 25 sequences, sampling the selected actions from the test set with replacement, whereas the GPs are learned from the training set. On top of that we repeat the crossvalidation for 10 times after shuffling randomly the data.

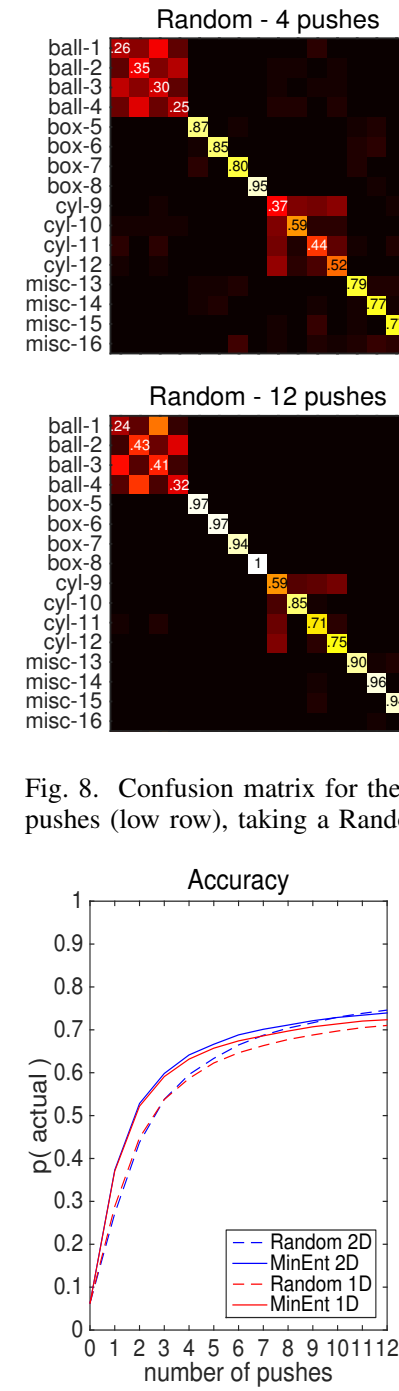

(a)
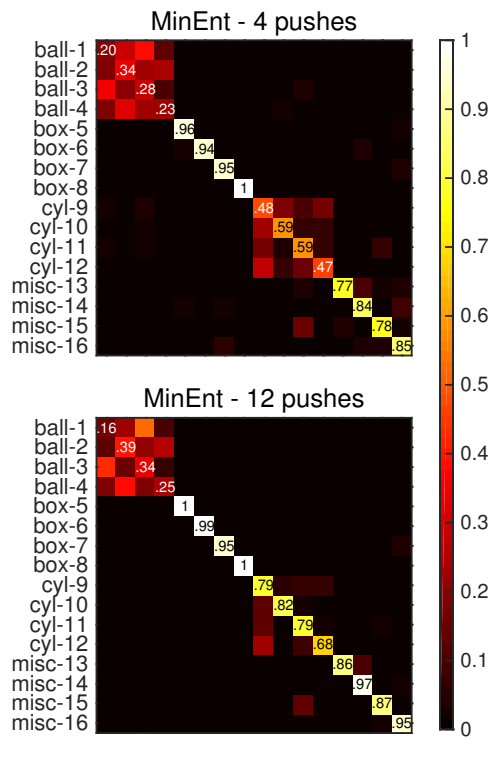

Fig. 8. Confusion matrix for the 16 objects after 4 pushes (top row) and 12 shes (low row), taking a Random (left) or MinEnt policy (right).

Fig. 9. (a) Average probability to recognize the actual object, taking a Random or MinEnt policy, for the 2D (blue) or 1D (red) action space. (b) Minimum number of pushes to reach a confidence of $80 \%$.

Fig. 8 presents the confusion matrices after 4 and 12 pushes in sequence, averaged over 500 iterations for each object.

After these sequences of pushes, the result of the classification looks similar in both cases, though MinEnt performs slightly better. The boxes and miscellaneous objects are classified correctly according to their instance, whereas the balls and the cylinders are confused within their respective groups. This can be noticed especially for the balls (upper left block). We would expect such a confusion, due to similarities in object shapes and the nature of our feature space. The confusions come from low-discriminative features allowing for generalization, also captured properly through the uncertainty in the trained models (see Fig. 7 for balls and rotation feature with cylinders). We will see later how the objects can be categorized based on these similarities.

To better see the effect of the action policy, we measure the average posterior probabilities of the actual class given by (5), with classes defined by the original instances of objects. The results shown in Fig. 9a illustrate the benefit of the entropy- 

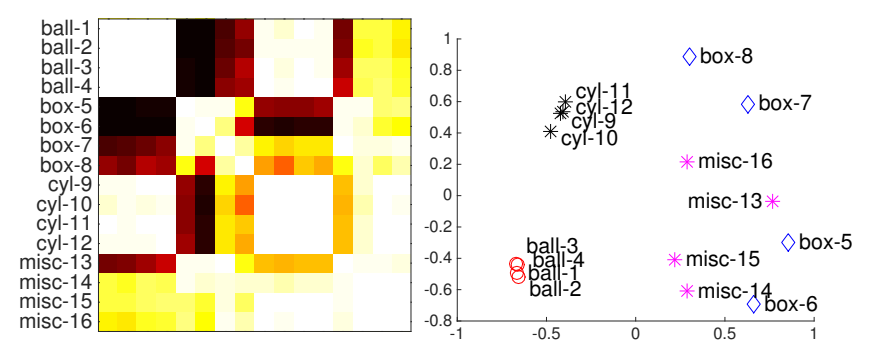

Fig. 10. Similarity matrix (left) based on a symmetric distance measure of the KL-divergence and the resulting spectral embedding (right).
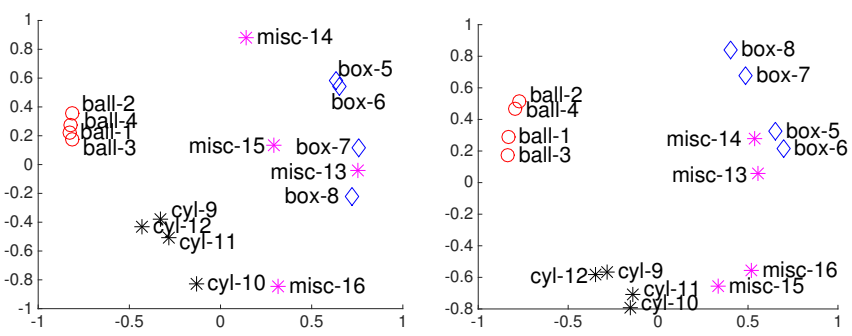

Fig. 11. Spectral embedding obtained from a noisy version of the training data, adding Gaussian noise with $\sigma_{n}^{2}=5$ (left) and $\sigma_{n}^{2}=10$ (right).

based selection where the actual classes are found with slightly higher posterior probabilities using MinEnt policy, especially with the first pushes. In [30] our action space was defined only by the position of the push. We compare this $1 \mathrm{D}$ action space, updated with the new objects, against the 2D action space defined by position and velocity. The slight benefit of MinEnt is similar with the new dimension but these differences tend to disappear when performing more pushes. We expected a clearer benefit with MinEnt as the action space becomes larger. Our interpretation is that every action is still informative regarding the actual class if the instance can be individuated.

As this result is an average over all the classes, it can also be hard to interpret depending on the category. In Fig. 9b we measure the minimum number of pushes to reach a confidence of $80 \%$ to detect each instance. This shows that boxes and miscellaneous objects are usually identified after 1 to 5 pushes with MinEnt, whereas 1 to 2 more pushes are required with Random. For balls and most of cylinders, the confusion within the category does not allow to reach the target confidence.

\section{Unsupervised categorization}

To regroup the objects into categories, we need a criterion measuring how similar two objects are. As the distributions are Gaussians, we compute the Kullback-Leibler divergence of two objects $i$ and $j$ given each feature and action. A symmetric distance measure is then derived as $d_{i j}=K L(i, j)+K L(j, i)$, that we average over the features and actions using the GPs trained on the full dataset. We define a similarity measure $S_{i j}=\exp \left(-d_{i j}^{2} / 2 \sigma_{d}^{2}\right)$ where $\sigma_{d}^{2}$ is the variance of the distance, giving the similarity matrix shown to the left in Fig. 10.

From this, we build a spectral embedding using the Normalized Cuts algorithm [43]. The result is shown to the right in Fig. 10. The balls and the cylinders form two dense clusters, whereas the box and the miscellaneous objects are individually separated with a higher spread. Those instances are specific

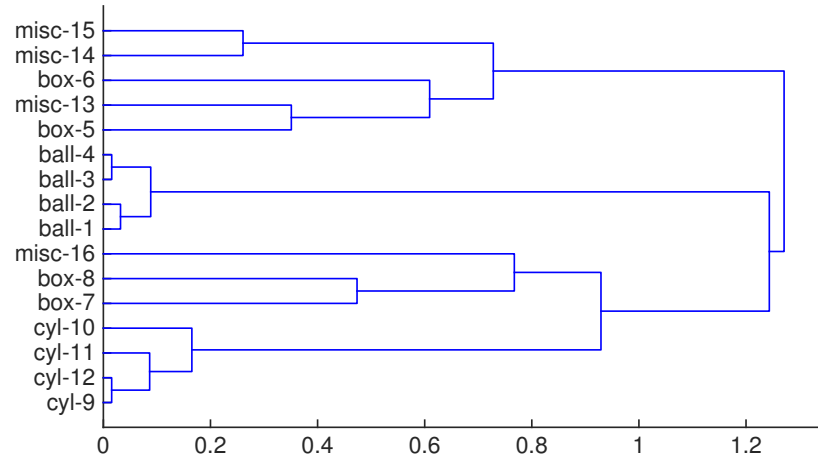

Fig. 12. Agglomerative clustering obtained from a complete-linkage of the spectral embedding, forming the object categories as a bottom-up hierarchy.
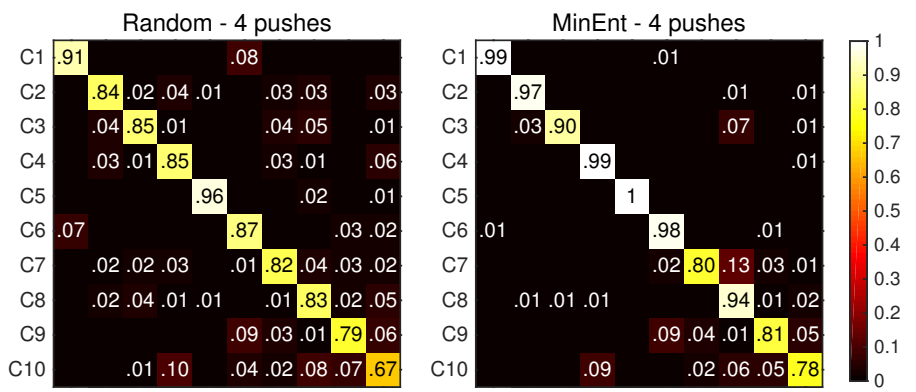

Fig. 13. Confusion matrix for 10 categories after 4 pushes, taking a Random (left) or MinEnt policy (right).
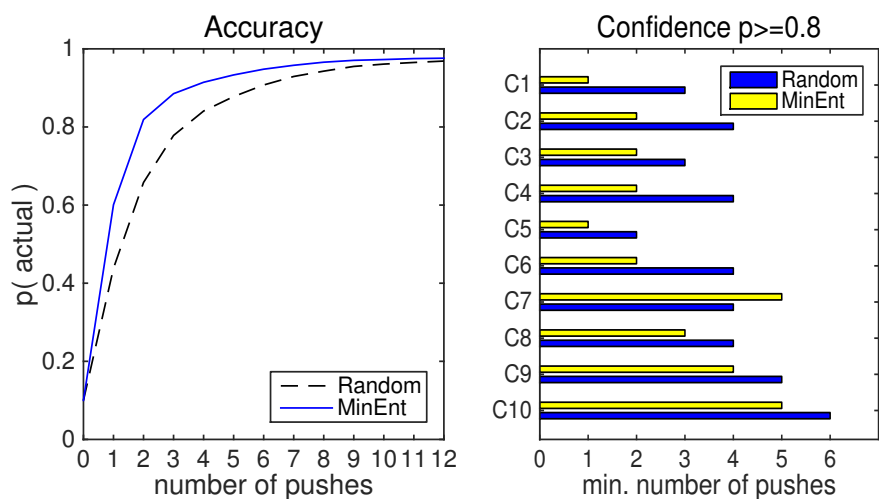

Fig. 14. Average probability to recognize the actual category taking a Random or MinEnt policy; minimum number of pushes to reach a confidence of $80 \%$.

though we expected a better generalization from the lowdiscriminative features. This result differs from [30] where the boxes presented more similarities. Our interpretation is that the pushes were performed much more regularly, now that the effective push is controlled with the motion detection. As a consequence, the training data of such objects shows very slight variations, represented accurately by the GP with a very low variance.

The high variance of the rotation feature for the balls and cylinders (no physical plane) then dominate the final result. This can be seen as a form of overfitting where the optimization of the hyperparameters of the GP leads to model each instance too specifically. This might however be a desired result, depending on the classification problem. To see if a better generalization can be obtained, we repeat the whole 
learning process with additive Gaussian noise in the training data. The effect is shown in Fig. 11 where the boxes are much closer, similarly to our previous work [30].

By performing an agglomerative clustering from pair-wise distances (from the original embedding in Fig. 10), we regroup the objects into categories, illustrated by the dendrogram in Fig. 12. The clusters are initialized with each instance and gradually composed by merging the closest clusters. The distance measure between a pair of clusters can be defined with different methods. We exclude the single-linkage, or nearest neighbour, causing the chaining phenomenon. Instead, we adopt the complete-linkage also known as farthest neighbour, leading to compact clusters of similar size.

New GP models are trained by merging the samples of objects belonging to the same category. The framework is used similarly, with the same cross-validation method, but here the classes represent the resulting categories instead of the object instances. As an example we create 10 categories by merging the 6 first levels in the hierarchical clustering, corresponding to balls and cylinders. The results of the classification in Fig. 13 and 14 show that already after 4 pushes all of the instances belonging to the category are recognized with a high confidence using MinEnt policy, which performs significantly better than Random.

\section{CONCLUSiOn AND Future WORK}

We presented an action-driven framework for sensorimotor learning and functional categorization of objects. The capability of the model is illustrated with a pushing behavior, represented by a 2-dimensional action space. By observing the effects of different actions applied to objects, sensorimotor contingencies were learned using a probabilistic model based on Gaussian Processes with an explicit representation of the effect. The possibility of making predictions with a measure of confidence was tested for classification, evaluating the entropy to select suitable actions. The presented experiments show the benefit of following such a policy. By looking at the similarities of SMCs obtained for different objects, categories were discovered and learned with the same model.

The key of this approach resides in the object-centered representation of the SMCs. This assumes the action space can be defined in such a way for the behavior of interest. If this was intuitive for the pushing, involving a visual sense, it might be difficult to define for other cases. In other words the condition is to define the effects in a symbolic representation where the action is not dependent of the current state.

We studied the effects of actions applied on objects for a single behavior, with simple features and limitations in the time domain. If the features associated with different behaviors are of similar nature it would be possible to define a global action space by concatenation. However it has to be studied how to combine them in the general case, for instance with a unified categorization [13], along with effects observed in the spatiotemporal domain such as done in [35].

The tasks of classification and categorization were driven exclusively by action. The environment was not modelled and the context associated with each SMC was limited to the instance of object regardless of attributes such as appearance. In an extension of this framework it could be investigated how to take the environment or static features into account.

Though we used low-distinctive features for the sake of generalization, the experiments showed that several instances were recognized individually, which is not necessarily desirable. We showed that additive noise in the training data helped to cancel this effect. This illustrates the risk of overfitting and raises the question about model selection and optimization of hyperparameters. In future studies it would be interesting to control the level of generalization in the training stage or in the similarity measure.

When it comes to the learning process, each object was explored randomly and the effect features were acquired for each instance through supervised learning. In future works different exploratory behaviors could be compared to better drive the learning stage. Instead of doing the categorization a posteriori, this could be done directly from the observations without labeling the classes, in an unsupervised fashion. The features were also assumed to be independent, while in reality they are not. It remains to be studied whether such a simplification is in fact justified, e.g. by training multi-output GP.

\section{ACKNOWLEDGMENT}

This work was supported by the EU through the project socSMCs (EC H2020-FETPROACT-2014 641321) and the Swedish Research Council. The authors would like to thank Prof. Emer. Jan-Olof Eklundh for his advices.

\section{REFERENCES}

[1] F. G. Ashby and W. T. Maddox, "Human category learning," Annи Rev Psychol, vol. 56, pp. 149-178, 2005.

[2] B. Leibe and B. Schiele, "Analyzing appearance and contour based methods for object categorization," in IEEE CVPR, 2003.

[3] G. Bouchard and B. Triggs, "Hierarchical part-based visual object categorization," in IEEE CVPR, pp. 710-715, 2005.

[4] S. Savarese and L. Fei-Fei, "3D generic object categorization, localization and pose estimation," in IEEE ICCV, pp. 1-8, 2007.

[5] V. Kruger, D. Kragic, A. Ude, and C. Geib, "The meaning of action: a review on action recognition and mapping," Advanced Robotics, vol. 21, no. 13 , pp. 1473-1501, 2007.

[6] H. Kjellström, J. Romero, and D. Kragic, "Visual object-action recognition: Inferring object affordances from human demonstration," Computer Vision and Image Understanding, vol. 115, no. 1, pp. 81-90, 2011.

[7] K. M. Varadarajan and M. Vincze, "Parallel Deep Learning with Suggestive Activation for Object Category Recognition," in ICVS, pp. 354-363, Springer-Verlag, 2013.

[8] J. Sinapov and A. Stoytchev, "From acoustic object recognition to object categorization by a humanoid robot," Workshop on Mobile Manipulation, Robotic Science and Systems conference, 2009.

[9] N. Dag, I. Atil, S. Kalkan, and E. Sahin, "Learning Affordances for Categorizing Objects and Their Properties," ICPR, pp. 3089-3092, 2010.

[10] E. Ugur, E. Oztop, and E. Sahin, "Goal emulation and planning in perceptual space using learned affordances," Robotics and Autonomous Systems, vol. 59, pp. 580-595, July 2011.

[11] J. Sinapov, V. Sukhoy, R. Sahai, and A. Stoytchev, "Vibrotactile Recognition and Categorization of Surfaces by a Humanoid Robot," IEEE Transactions on Robotics, vol. 27, no. 3, pp. 488-497, 2011.

[12] J. Sinapov, C. Schenck, K. Staley, V. Sukhoy, and A. Stoytchev, "Grounding semantic categories in behavioral interactions: Experiments with 100 objects," Robotics and Autonomous Systems, Nov. 2012.

[13] S. Griffith, J. Sinapov, V. Sukhoy, and A. Stoytchev, "A BehaviorGrounded Approach to Forming Object Categories: Separating Containers From Noncontainers," IEEE TAMD, vol. 4, no. 1, pp. 54-69, 2012. 
[14] J. K. O'Regan and A. Noë, "A sensorimotor account of vision and visual consciousness," Behavioral and Brain Sciences 24(5), pp. 9391031, 2001.

[15] A. Rebguns, D. Ford, and I. R. Fasel, "InfoMax Control for Acoustic Exploration of Objects by a Mobile Robot," Workshops at the TwentyFifth AAAI Conference on Artificial Intelligence, 2011.

[16] J. A. Fishel and G. E. Loeb, "Bayesian exploration for intelligent identification of textures," Frontiers in Neurorobotics, vol. 6, p. 4, 2012

[17] S. Ivaldi, S. M. Nguyen, N. Lyubova, A. Droniou, V. Padois, D. Filliat, P. Y. Oudeyer, and O. Sigaud, "Object Learning Through Active Exploration," IEEE TAMD, vol. 6, no. 1, pp. 56-72, 2014.

[18] J. J. Gibson, The Ecological Approach to Visual Perception. Psychology Press, 1979.

[19] P. Fitzpatrick, G. Metta, L. Natale, S. Rao, and G. Sandini, "Learning about objects through action - initial steps towards artificial cognition," in IEEE ICRA, pp. 3140-3145, 2003.

[20] M. Sanchez-Fibla, A. Duff, and P. F. M. J. Verschure, "The acquisition of intentionally indexed and object centered affordance gradients: A biomimetic controller and mobile robotics benchmark," in IEEE/RSJ IROS, pp. 1115-1121, 2011.

[21] L. Montesano, M. Lopes, A. M. Bernardino, and J. Santos-Victor, "Learning Object Affordances: From Sensory-Motor Coordination to Imitation," IEEE Transactions on Robotics, vol. 24, no. 1, pp. 15-26, 2008.

[22] K. M. Varadarajan and M. Vincze, "Affordance based part recognition for grasping and manipulation," Workshop on Autonomous Grasping, IEEE ICRA, 2011

[23] A. Aldoma, F. Tombari, and M. Vincze, "Supervised learning of hidden and non-hidden 0-order affordances and detection in real scenes," in IEEE ICRA, pp. 1732-1739, 2012.

[24] F. Guerin, N. Kruger, and D. Kraft, "A Survey of the Ontogeny of Tool Use: From Sensorimotor Experience to Planning," IEEE TAMD, 2013.

[25] A. Stoytchev, "Behavior-Grounded Representation of Tool Affordances," in IEEE ICRA, pp. 3071-3076, 2005.

[26] J. Sinapov and A. Stoytchev, "Detecting the functional similarities between tools using a hierarchical representation of outcomes," in IEEE ICDL, pp. 91-96, 2008

[27] D. M. Wolpert and M. Kawato, "Multiple paired forward and inverse models for motor control," Neural Networks, vol. 11, Oct. 1998.

[28] M. Haruno, D. M. Wolpert, and M. Kawato, "MOSAIC Model for Sensorimotor Learning and Control," Neural Computation, vol. 13, pp. 2201-2220, Oct. 2001.

[29] R. Pfeifer, J. Bongard, and S. Grand, How the Body Shapes the Way We Think. A New View of Intelligence, MIT Press, 2007.

[30] V. Högman, M. Björkman, and D. Kragic, "Interactive object classification using sensorimotor contingencies," in IEEE/RSJ IROS, pp. 2799$2805,2013$.

[31] D. Nguyen-Tuong and J. Peters, "Model learning for robot control: a survey," Cogn Process, vol. 12, pp. 319-340, Apr. 2011.

[32] C. E. Rasmussen and C. K. I. Williams, Gaussian processes for machine learning. MIT Press, 2006.

[33] A. Stoytchev, "Some Basic Principles of Developmental Robotics," IEEE TAMD, vol. 1, no. 2, pp. 122-130, 2009.

[34] N. Krüger, C. Geib, J. Piater, R. Petrick, M. Steedman, F. Wörgötter, A. Ude, T. Asfour, D. Kraft, D. Omrčen, A. Agostini, and R. Dillmann, "Object-Action Complexes: Grounded abstractions of sensory-motor processes," Robotics and Autonomous Systems, vol. 59, pp. 740-757, Oct. 2011

[35] E. E. Aksoy, A. Abramov, J. Dorr, K. Ning, B. Dellen, and F. Wörgötter, "Learning the semantics of object-action relations by observation," The International Journal of Robotics Research, vol. 30, pp. 1229-1249, Sept. 2011.

[36] A. Duff, M. Sanchez-Fibla, and P. F. M. J. Verschure, "A biologically based model for the integration of sensory-motor contingencies in rules and plans: A prefrontal cortex based extension of the Distributed Adaptive Control architecture," Brain Research Bulletin, vol. 85, pp. 289-304, 2011.

[37] G. Metta and P. Fitzpatrick, "Better Vision through Manipulation," Adaptive Behavior, pp. 109-128, 2003.

[38] A. Maye and A. K. Engel, "A discrete computational model of sensorimotor contingencies for object perception and control of behavior," IEEE ICRA, 2011.

[39] B. Ridge and A. Ude, "Action-grounded push affordance bootstrapping of unknown objects," in IEEE/RSJ IROS, pp. 2791-2798, 2013.

[40] M. Fischler and R. Bolles, "Random sample consensus: A paradigm for model fitting with applications to image analysis and automated cartography," Communications of the ACM, vol. 24(6), pp. 381-395, 1981.

[41] M. Björkman and D. Kragic, "Active 3D scene segmentation and detection of unknown objects," IEEE ICRA, 2010.

[42] G. Farnebäck, "Two-frame motion estimation based on polynomial expansion," in SCIA'03: Proceedings of the 13th Scandinavian conference on Image analysis, Springer-Verlag, June 2003.

[43] J. Shi and J. Malik, "Normalized cuts and image segmentation," IEEE TPAMI, vol. 22, no. 8, pp. 888-905, 2000 .

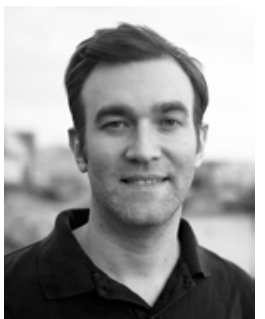

Virgile Högman received the M.Sc. degree in computer science from Paul Sabatier University of Toulouse, France, in 1997, and the M.Sc. degree in computer science and engineering from the Royal Institute of Technology (KTH), Stockholm, Sweden, in 2011.

During 1997-2009 he worked as software engineer and technical manager in the aerospace industry, in France and Italy. His previous work concerned systems design and integration, ground segment operations of observation satellites. $\mathrm{He}$ is currently working towards a Ph.D. degree in computer science at KTH. His current research interests include autonomous and developmental robotics, cognitive systems, computer vision, and machine learning.

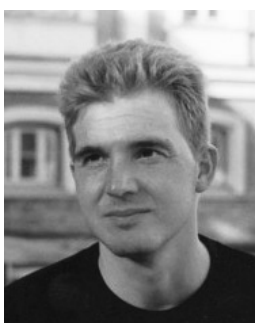

Mårten Björkman received the M.Sc. degree in computer science and engineering from Lund University, Sweden in 1994 and the Ph.D. in computer science from KTH in 2002.

$\mathrm{He}$ is currently an Associate Professor at the School of Computer Science and Communication at KTH in Stockholm. In 2005 he co-founded Intelligent Mobile Documentation, a company in the automatic ticketing business. His research is in the areas of computer vision, robotics, machine learning and cognition, with recent focus on self-learning of autonomous systems with multi-modal sensing. Other interests involve largescale parallel computer architectures for real-time processing and learning from visual data.

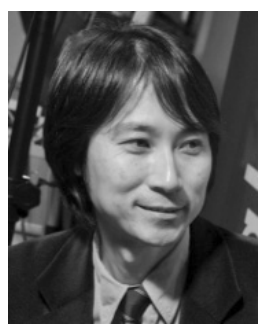

Atsuto Maki received the $\mathrm{Ph} . \mathrm{D}$. degree in computing science from the Royal Institute of Technology (KTH), Stockholm, Sweden, in 1996. Prior to that he studied electrical engineering at Kyoto University and the University of Tokyo, Japan.

$\mathrm{He}$ is currently an Associate Professor at the School of Computer Science, KTH. He has previously been a Senior Researcher at Toshiba Research Europe, Cambridge, UK, and an Associate Professor at the Graduate School of Informatics, Kyoto University, and a Research Scientist at Toshiba's Corporate R\&D Center. His recent research interests include machine learning and computer vision, motion and object recognition, clustering, subspace analysis, and representation learning. He has been serving as a program committee member at major computer vision conferences, including as an Area Chair of ICCV'09.

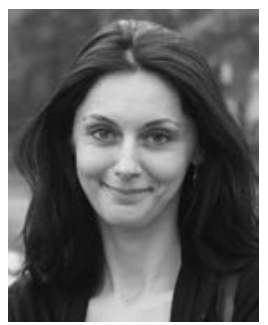

Danica Kragic received the M.Sc. degree in mechanical engineering from the Technical University of Rijeka, Croatia in 1995, and the Ph.D. degree in computer science from KTH, Stockholm, in 2001.

She is currently a Professor at the School of Computer Science and Communication at KTH. Her research is in the area of computer vision, object grasping and manipulation and human-robot interaction. Her recent work explores different learning methods for formalizing the models for integrated representation of objects and actions that can be applied on them. This work has demonstrated how robots can achieve scene understanding through active exploration and how full body tracking of humans can be made more efficient.

Danica received the 2007 IEEE Robotics and Automation Society Early Academic Career Award. She is a member of the Swedish Royal Academy of Sciences and Swedish Young Academy. 